\title{
Osteoma de oído medio asintomático
}

\section{Asymptomatic middle ear osteoma}

\author{
Andrés Caballero G. ${ }^{1}$, Laura Riera T. ${ }^{1}$, Dolores Alonso B. ${ }^{2}$
}

Servicio de Otorrinolaringología, Hospital Universitario Puerta del Mar. Cádiz, España. ${ }^{2}$ Servicio de Otorrinolaringología, Hospital Universitario Clínico San Cecilio. Granada, España.

Los autores declaran no tener conflictos de interés.

Recibido el 1 de diciembre de 2020. Aceptado el 11 de enero de 2021

Correspondencia: Andrés Caballero $\mathrm{G}$ Avda Ana de Viya, № 21

CP: 11009

Hospital Universitario Puerta del Mar Servicio de Otorrinolaringología. Cádiz, España. Email: handdres@gmail.com

\section{Resumen}

El osteoma de oído medio es un tumor poco común, de crecimiento lento y características benignas. Frecuentemente cursa de forma asintomática y su diagnóstico suele ser casual como en el caso que exponemos. Se presenta el caso de un varón de 26 años, que consultó por odinofagia y otalgia derecha en el contexto de una infección de vía aérea alta. Al explorarlo mediante otoscopia se visualizó en el oído izquierdo una lesión blanquecina y redondeada retrotimpánica compatible con osteoma de oído medio. El tratamiento de elección en pacientes con osteomas de oído medio sintomáticos es la cirugía. Sin embargo, en pacientes asintomáticos el seguimiento es la opción más recomendada.

Palabras clave: Oído medio, osteoma, pérdida auditiva conductiva, hueso temporal, tomografía computada.

\begin{abstract}
The middle ear osteoma is a benign, infrequent, slow-growing tumor. This lesion is frequently asymptomatic and its diagnosis is usually accidental, as the case presented here. We present a case of a 26-year-old man who consulted for odynophagia and right ear pain in the context of an upper airway infection. By otoscopy in the left ear, we observed a whitish retrotympanic lesion that was compatible with a middle ear osteoma. To manage symptomatic patients with middle ear osteomas, surgery is the treatment of first choice. Nevertheless, for asymptomatic patients, follow up is recommended.

Keywords: Middle ear, osteoma, conductive hearing loss, temporal bone, computed tomography.
\end{abstract}

\section{Introducción}

Los osteomas son tumoraciones benignas muy frecuentes en el hueso temporal. Su localización habitual suele ser en el conducto auditivo externo (CAE) y, aunque existen distintas teorías (genética, inflamatoria, etc.), son de etiología desconocida ${ }^{1}$. Los osteomas se presentan como lesiones unilaterales, redondeadas, únicas y pediculadas; y suelen localizarse en el área más externa del CAE óseo. Hay que diferenciarlos de otras lesiones comunes conocidas como exóstosis: son masas planas, elevaciones de hueso no tumorales; de base ancha, bilaterales y de situación más profunda en el CAE. La etiología de las exóstosis es conocida y está claramente relacionada con la exposición continuada al agua fría ${ }^{2}$; de hecho, las exóstosis son vulgarmente conocidas como "oído de surfista".

Los osteomas también pueden hallarse en el oído medio, aunque esta situación es mucho menos frecuente ${ }^{1}$. El síntoma más habitual de los osteomas de oído medio es la hipoacusia de conducción $(69,4 \%)$, aunque frecuentemente son asintomáticos $(16,7 \%)$ y su diagnóstico es incidental ${ }^{2}$.

\section{Caso Clínico}

Varón de 26 años, sin antecedentes personales de interés, que consulta por odinofagia y otalgia derecha de tres días de evolución en el contexto de un cuadro catarral. En la exploración física observamos la faringe eritematosa 
y la otoscopia del oído derecho sin alteraciones. En el oído izquierdo se objetivó una lesión blanquecina retrotimpánica a nivel de promontorio y cercana al mango del martillo. Dada la situación de la lesión, se sospechó que pudiera tratarse de un colesteatoma secundario a la colocación de un drenaje transtimpánico, cirugía o tras trauma directo de la membrana timpánica. Sin embargo, el paciente no presentaba antecedentes previos de este tipo y su membrana timpánica era totalmente traslúcida, sin evidencia de miringoesclerosis ni cicatrices. Dado que tampoco había padecido otitis de repetición en la infancia, se sospecha entonces de un colesteatoma congénito y se solicita una tomografía computarizada (TC) de oídos (Figuras 1 y 2).

En el TC se aprecia una lesión de densidad calcio, bien delimitada en el oído medio, apoyada sobre el promontorio que mide $1 \times 1,4$ $\mathrm{mm}$ de diámetro en los planos transversal y anteroposterior. La audiometría tonal mostraba umbrales auditivos dentro de la normalidad. Se decide exploración bajo anestesia local y mirigotomía simple; palpándose la lesión que presenta una consistencia dura, fija y no móvil. Dada la localización de la lesión y que el paciente se encontraba asintomático, se propone seguimiento, continuando asintomático al día de hoy.

\section{Discusión}

Yoon y cols. ${ }^{2}$ publicaron un artículo en 2014 donde, además de presentar dos casos de osteoma en oído medio, realizaron una revisión de 34 casos publicados con anterioridad (en total 36 casos). Llegaron a la conclusión de que el osteoma de oído medio aparecía el doble en varones que en mujeres 2:1 (otros autores como Abouzayad en su publicación de 2015, baja esta proporción a 1,75:1 varón/ mujer ${ }^{3}$ ), siendo más frecuente en pacientes jóvenes (media de 28,5 años), siendo el síntoma principal la hipoacusia transmisiva $(67,6 \%)$. Dependiendo de su localización pueden presentar otros síntomas, aunque son menos frecuentes: síntomas vestibulares, parálisis facial, otorrea, secreción o acúfeno. Aquellos

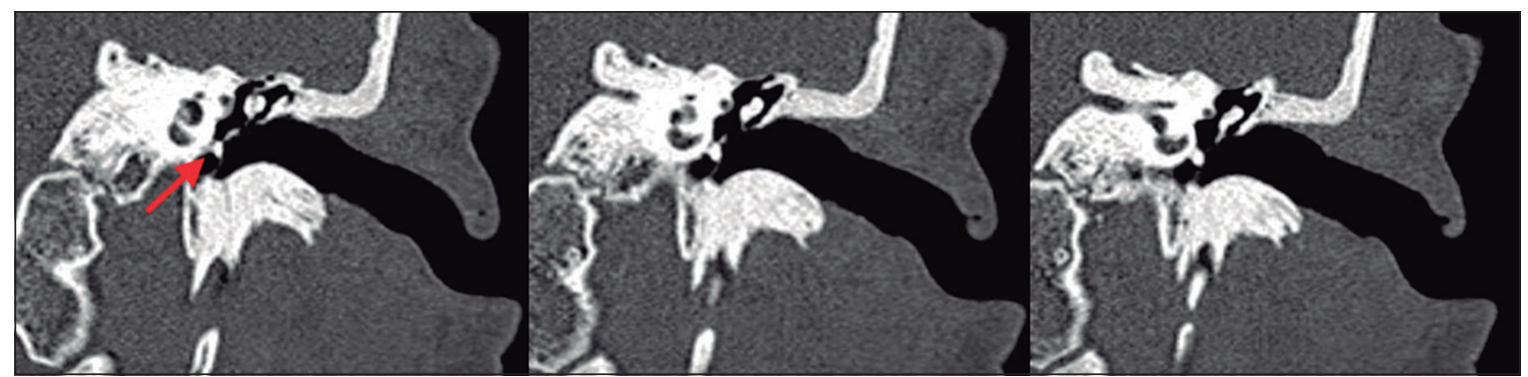

Figura 1. Tomografía computada de oídos, cortes coronales seriados del oído izquierdo. Se aprecia el osteoma de oído medio sobre el promontorio (flecha).

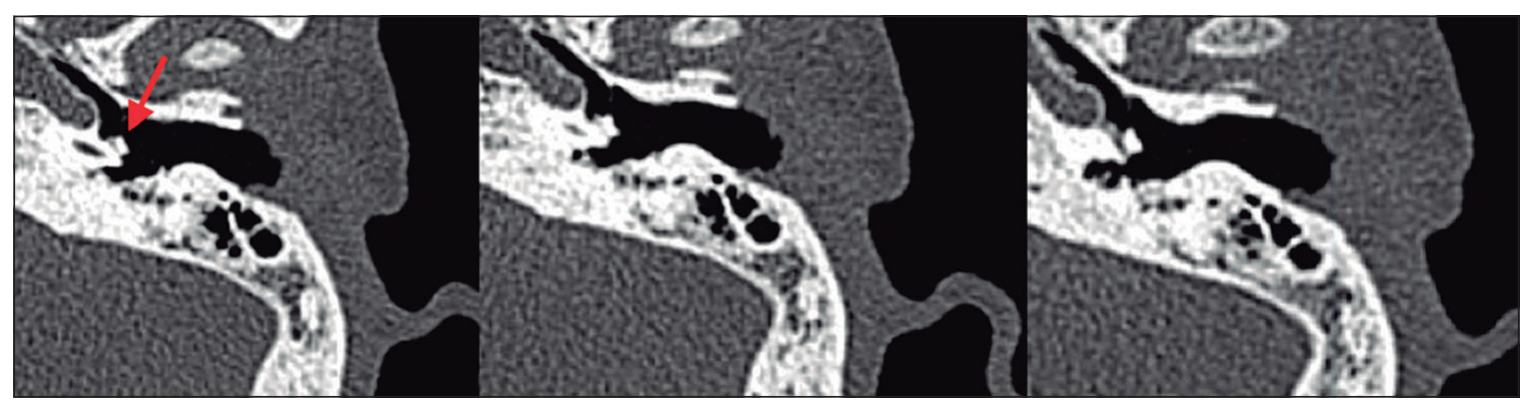

Figura 2. Tomografía computarizada de oídos, cortes axiales seriados del oído izquierdo. Se visualiza el osteoma sobre el promontorio (flecha). 
osteomas que no afectan a la cadena osicular, suelen cursar como asintomáticos. Se localizan mayoritariamente en el promontorio $(33,3 \%)$, yunque $(13,9 \%)$, proceso piramidal $(11,1 \%)$ y epitímpano $(11,1 \%)^{2}$.

Puesto que el artículo de Yoon y cols. ${ }^{2}$ se publicó en 2014, planteamos la actualización de su base de datos. Para ello realizamos una búsqueda en PUBMED mediante los MESH ("Ear, Middle"[Mesh]) y ("Osteoma”[Mesh]) con el operador booleano AND y restringiendo la fecha de publicación desde el año 2013 a la actualidad (noviembre del año 2020). Se localizan 10 artículos, de los cuales 8 de ellos exponen casos clínicos de osteomas en oído medio no incluidos en la revisión de Yoon y cols. ${ }^{2}$, y una de estas publicaciones presenta 2 pacientes. Se completa la búsqueda lanzándola nuevamente con el texto simple "Ear, Middle AND Osteoma" eliminando las publicaciones obtenidas en la búsqueda anterior con el operador booleano NOT [NOT ("Ear, Middle”[Mesh] AND “Osteoma”[Mesh])] y restringiéndola a la misa fecha. Se localizan 26 publicaciones más, de las cuales sólo 4 presentan nuevos casos de osteomas en oído medio. En total, incluyendo nuestro caso, se añaden 14 pacientes más a la base de datos creada por
Yoon y cols. ${ }^{2}$ (Tabla 1).

Se estudian datos biométricos (edad y localización de la lesión) además del síntoma principal. Se realiza análisis de los datos mediante el paquete estadístico SPSS V.25. Estudiamos a 50 pacientes, 22 mujeres (44\%) y 28 varones $(56 \%)$. La edad oscila entre los 4-57 años, con una media de 29,54 años y una mediana en 28,5 años; el 75\% de los pacientes eran menores de 42 años. En el $40 \%$ de los casos la lesión se situaba en el promontorio, el $12 \%$ en el epitímpano, el $12 \%$ en el yunque y el $8 \%$ en el proceso piramidal. La hipoacusia de transmisión es el síntoma más frecuente y aparece en el $62 \%$ de los casos, seguida de los asintomáticos que son el 18\% de los casos y la hipoacusia mixta que la presentan el 14\% de los pacientes estudiados.

Hay que destacar que, al analizar los datos tras actualizar la base, la proporción varones/ mujeres es de 1,3:1 en vez de 2:1 como había publicado Yoon y cols. ${ }^{2}$ en su estudio. El resto de los parámetros no presentaban cambios importantes.

El tratamiento de elección para el osteoma de oído medio es la resección quirúrgica. El oído medio es una cavidad de pequeño tamaño (habitualmente oscila entre 1-1,5 centíme-

\begin{tabular}{|llllll|}
\hline \multicolumn{7}{l}{ Tabla 1. Osteomas de oído medio en la literatura } & & \\
\hline Autor & Sexo & Edad & Fecha & Localización & Síntoma \\
\hline Yuan et al. ${ }^{4}$ & Mujer & 38 & 2013 & Estribo & Hipoacusia mixta \\
\hline Sáez et al. ${ }^{5}$ & Mujer & 36 & 2014 & Promontorio & Ninguno \\
\hline Toro et al. $^{6}$ & Mujer & 33 & 2014 & Promontorio & Hipoacusia de transmisión \\
\hline Chang et al. ${ }^{7}$ & Mujer & 14 & 2014 & Promontorio & Hipoacusia de transmisión \\
\hline Curtis et al. ${ }^{8}$ & Mujer & 49 & 2014 & Hipotímpano & Hipoacusia de transmisión \\
\hline Park et al. ${ }^{9}$ & Mujer & 4 & 2014 & Promontorio & Ninguno \\
\hline Abouzayd et al. ${ }^{3}$ & Mujer & 56 & 2015 & Epitímpano & Hipoacusia de transmisión \\
\hline Molher et al. ${ }^{1}$ & Varón & 23 & 2018 & Promontorio & Hipoacusia mixta \\
\hline Garov et al. ${ }^{10}$ & Mujer & 29 & 2019 & Yunque & Hipoacusia mixta \\
\hline Garov et al. ${ }^{10}$ & Varón & 31 & 2019 & Epitímpano & Hipoacusia mixta \\
\hline Gülşen ${ }^{11}$ & Varón & 21 & 2019 & Promontorio & Hipoacusia de transmisión \\
\hline Saylisoy ${ }^{12}$ & Mujer & 53 & 2020 & Trompa de Eustaquio & Otitis media \\
\hline Falcioni et al. ${ }^{13}$ & Mujer & 36 & 2020 & Promontorio & Hipoacusia de transmisión \\
\hline Caballero et al. & Varón & 26 & 2021 & Promontorio & Ninguno \\
\hline
\end{tabular}


tros cúbicos en el adulto, y es aún más pequeño en los niños). Está en íntimo contacto con estructuras nobles cuya lesión podría perjudicar seriamente a la calidad de vida del paciente. Dependiendo de la localización en la que se encuentre la lesión, la intervención quirúrgica podría tener las siguientes complicaciones más frecuentes: hipoacusia y/o acúfeno (si se lesiona la cóclea), vértigo crónico (si afecta al vestíbulo o a los canales semicirculares, principalmente al canal semicircular lateral por ser el más expuesto), parálisis facial (por lesión del nervio facial en su segunda o tercera porción), fístula de líquido cefalorraquídeo (por lesión del tegmen tympani) o sangrado importante (por lesión de la arteria carótida interna o del bulbo de la vena yugular interna según asiente la lesión en la pared anterior o inferior de la caja timpánica). Dado el riesgo que presenta la cirugía, y que en un gran número de pacientes los osteomas de oído medio cursan asintomáticos; muchos otólogos optan por la observación y seguimiento de las lesiones, reservándose la cirugía para los casos más severos o sintomáticos. Park ${ }^{9}$ en un artículo publicado en 2014 refiere que si el osteoma de caja es asintomático no es necesaria su exéresis quirúrgica y recomienda un seguimiento periódico con exploración otoscópica, audiométrica y TC; y en caso de un crecimiento excesivo que produzca pérdida de audición, obstrucción de la trompa de Eustaquio o afectación de la cadena osicular, debería de considerarse la cirugía. Esta teoría de la actitud expectante es apoyada por autores como Molher ${ }^{1}$, Güls en ${ }^{11}$ o Marchioni ${ }^{14}$. Este último plantea distintos abordajes quirúrgicos dependiendo de la extensión de la lesión y la afectación de tejidos vecinos; así lesiones pequeñas y localizadas podrían ser intervenidas mediante abordaje transcanal, y otras mayores o con afectación de otras estructuras podrían precisar un abordaje retroauricular o incluso una mastoidectomía ${ }^{14}$.

La TC de alta resolución es la prueba de imagen más recomendada para el estudio de los osteomas de oído medio. La TC valora muy bien las estructuras óseas y por eso es muy útil para estudiar la situación y los límites de estos tumores, además de objetivar si existe afectación de la cadena osicular y/o del acueducto de Falopio y, por tanto, del nervio facial ${ }^{11}$.

\section{Conclusión}

El osteoma de oído medio parece ser más frecuente en varones que en mujeres. Hasta ahora se sospechaba que en una proporción 2:1 o 1,75:1; aunque tras la actualización de la base de datos presentada en este artículo esta proporción podría ser de hasta 1,3:1.

El osteoma de oído medio es más frecuente en pacientes jóvenes, suele aparecer más habitualmente en el promontorio y el síntoma que presentan los pacientes más frecuentemente es una hipoacusia de transmisión. La extirpación quirúrgica es el tratamiento de elección. Aunque parece adecuado mantener una actitud expectante en aquellos pacientes que no tengan síntomas; ya que, dadas las características y la situación de la lesión, la cirugía no está exenta de riesgos.

\section{Bibliografía}

1. Molher J, De Mones Del Pujol E, Do Santos Zounon A, Darrouzet V, Bonnard D. Middle ear osteoma causing mixed hearing loss: A case report. J Int $A d v$ Otol. 2018;14(3):493-6.

2. Yoon YS, Yoon YJ, Lee EJ. Incidentally detected middle ear osteoma: Two cases reports and literature review. Am J Otolaryngol. 2014;35(4):524-8. doi: 10.1016/j.amjoto.2014.03.010.

3. Abouzayd M, Seguir A. Epitympanic osteoma of the middle ear: A case report and literature review. Rev Larygol Otol Rhinol. 2015;136(2):81-3.

4. Yuan W, Chen L, Jiang X, Zhang X. Osteoma of stapes in the middle ear: A case report. Otol Neurotol. 2013;34(8):119-20.

5. Sáez Martínez ME, Rodríguez Sánchez D, Sánchez Jiménez RM. Osteoma del promontorio: Un hallazgo casual. Acta Otorrinolaringol Esp. 2014;65(1):64-5. doi: 10.1016/j.otorri.2012.05.006.

6. Toro PC, Castillo ÀC, Moya Martínez R, Domènech Juan I. Middle Ear Promontory Osteoma. Am J Otolaryngol. 2014;35(5):626-7. doi: 10.1016/j. amjoto.2014.07.005.

7. Chang TS, Lai W Sen, Kuo CY, Wang CH. Osteoma of the middle ear. Ear Nose Throat J. 2014;93(10):E43.

8. Curtis K, Bance M, Carter M, Hong P. Middle ear osteoma causing progressive facial nerve weakness: A case report. J Med Case Rep. 2014;8(1):1-3.

9. Park HJ, Eom TH, Cho YB, Jang CH. Osteoma of the promontory mimicking a congenital cholesteatoma. Korean J Audiol. 2014;18(1):38-40.

10. Garov E, Zelikovich E, Ivoylov A, Sidorina N, 


\section{CASO CLÍNICO}

Kaloshina A, Garova E. Middle ear osteoma: case report and literature review. Vestn Otorinolaringol. 2019;55-60.

11. Gülşen S. Promontory osteoma mimicking otosclerosis: Case report. J Laryngol Otol. 2019;133(12):1107-9.

12. Saylisoy S. Multiple Osteomas of Eustachian Tube Causing Ipsilateral Otitis Media. Curr Med Imaging.
2020;16(8):1052-4.

13. Falcioni M, Bertoli G, Ciavarro G. An uncommon cause of conductive hearing loss. Am J Otolaryngol. 2020;41(6). doi: 10.1016/j.amjoto.2020.102717.

14. Marchioni D, Alicandri-Ciufelli M, Gioacchini FM, Bonali M, Presutti L. Transcanal endoscopic treatment of benign middle ear neoplasms. Eur Arch Otorhinolaryngol. 2013;270(12):2997-3004. 